\title{
Pre-Ictal Phase Detection with SVMs
}

\author{
Julián R. Cózar ${ }^{1}$, Vesna Zeljković ${ }^{2}$, José Ma González-Linares ${ }^{1}$, \\ Nicolás Guil ${ }^{1}$, Milena Bojic ${ }^{3}$, Ventzeslav Valev ${ }^{4}$ \\ ${ }^{1}$ Computer Architecture Department, University of Málaga, Málaga, Spain
}

\author{
${ }^{2}$ School of Engineering \& Computing Sciences, New York Institute of Technology, Nanjing Campus, USA, vzeljkov@nyit.edu \\ ${ }^{3}$ University Clinic for Internal Diseases, Clinical Hospital Center "Dr Dragisa Misovic - Dedinje", Belgrade, Republic of Serbia \\ ${ }^{4}$ IAPR Fellow, School of Computing, University of North Florida, Florida, USA
}

\begin{abstract}
Over 50 million persons worldwide are affected by epilepsy. Epilepsy is a brain disorder known for sudden, unexpected transitions from normal to pathological behavioral states called epileptic seizures. Epilepsy poses a significant burden to society due to associated healthcare cost to treat and control the unpredictable and spontaneous occurrence of seizures. There is a need for a quick screening process that could help neurologist diagnose and determine the patient's treatment. Electroencephalogram has been traditionally used to diagnose patients by evaluating those brain functions that may correspond to epilepsy. The objective of this paper is to implement a novel detection technique of pre-ictal state that announces epileptic seizures from the online EEG data analysis. Unlike most published methods, that are aimed to distinguish only the normal from the epilepsy state, in this work the pre-ictal state is introduced as a new patient status, thus differentiating three possible states: normal (healthy), pre-ictal and epileptic seizure. In this manner, the patient should get timely alert about the possible seizure attack so that she/he can stop with its activities and take safety precautions.
\end{abstract}

EEG signal; Epilepsy; Pre-ictal; Classification; Wavelet Transform; SVM.

\section{REFERENCES}

[1] Dua T., De Boer H.M., Prilipko L.L., Saxena S.; Epilepsia, Epilepsy Care in the World: Results of an ILAE/IBE/WHO Global Campaign Against Epilepsy Survey, Vol. 47, Issue 7, pp. 1225-1231, July 2006.

[2] Mormann F., Andrzejak R., Elger C., Lehnertz K,; Seizure prediction: the long and winding road; Brain - Journal of Neurology (2007) 130 (2): 314-333.

[3] Fisher R., Saul M., "Overview of Epilepsy", Stanford Neurology.www.epilepsy.com/pdfs/Epilepsy-Handout.pdf

[4] Kalitzin S., Petkov G., Velis D., Vledder B., Lopes da Silva F., "Automatic Segmentation of Episodes Containing Epileptic Clonic Seizures in Video Sequences", IEEE Transactions on Biomedical Engineering, Dec. 2012, Vol. 59, Issue 12, pp. 3379 - 3385.

[5] Bhattacharyya S., Biswas A., Mukherjee J., Majumdar A. K., Majumdar B., Mukherjee S., Singh A. K., "Feature Selection for Automatic Burst Detection in Neonatal Electroencephalogram", IEEE Journal on Emerging and Selected Topics in Circuits and Systems, Dec. 2011, Vol. 1 , Issue 4, pp. 469 - 479.

[6] Chaovalitwongse W.A., Pottenger R.S., Shouyi Wang, Ya-Ju Fan, Iasemidis L.D., "Pattern- and Network-Based Classification Techniques for Multichannel Medical Data Signals to Improve Brain Diagnosis", IEEE Transactions on Systems, Man and Cybernetics, Part A: Systems and Humans, Sept. 2011, Vol. 41, Issue 5, pp. $977-$ 988.

[7] Chisci L., Mavino A., Perferi G., Sciandrone M., Anile C., Colicchio G., Fuggetta F., "Real-Time Epileptic Seizure Prediction Using AR Models and Support Vector Machines", IEEE Transactions on Biomedical Engineering, May 2010, Vol. 57, Issue 5, pp. $1124-$ 1132.

[8] Verma N., Shoeb A., Bohorquez J., Dawson J., Guttag J., Chandrakasan, A.P., "A Micro-Power EEG Acquisition SoC with Integrated Feature Extraction Processor for a Chronic Seizure Detection System", IEEE Journal of Solid-State Circuits, April 2010, Vol. 45, Issue 4, pp. $804-816$.

[9] Tzallas A.T., Tsipouras M.G., Fotiadis D.I., "Epileptic Seizure Detection in EEGs Using Time-Frequency Analysis", IEEE Transactions on Information Technology in Biomedicine, Sept. 2009, Vol. 13, Issue 5, pp. $703-710$.

[10] Van Hese P., Martens J. P., Waterschoot L., Boon P., Lemahieu I., "Automatic Detection of Spike and Wave Discharges in the EEG of Genetic Absence Epilepsy Rats from Strasbourg", IEEE Transactions on Biomedical Engineering, March 2009, Vol. 56, Issue 3, pp. $706-$ 717.

[11] Ghosh-Dastidar S., Adeli H., Dadmehr N., "Principal Component Analysis-Enhanced Cosine Radial Basis Function Neural Network for Robust Epilepsy and Seizure Detection", IEEE Transactions on Biomedical Engineering, Feb. 2008, Vol. 55, Issue 2, pp. $512-518$.

This work is partially supported by the Ministry of Education and Science of Spain under contract TIN2010-16144 and Junta de Andalucía under contract TIC-1692 
[12] Adeli H., Ghosh-Dastidar S., Dadmehr N., "A Wavelet-Chaos Methodology for Analysis of EEGs and EEG Subbands to Detect Seizure and Epilepsy", IEEE Transactions on Biomedical Engineering, Feb. 2007, Vol. 54, Issue 2, pp. 205 - 211.

[13] Chaovalitwongse W.A., Ya-Ju Fan, Sachdeo R.C., "On the Time Series K-Nearest Neighbor Classification of Abnormal Brain Activity", IEEE Transactions on Systems, Man and Cybernetics, Part A: Systems and Humans, Nov. 2007, Vol. 37, Issue 6, pp. $1005-$ 1016.

[14] Firpi H., Goodman E.D., Echauz J., "Epileptic Seizure Detection Using Genetically Programmed Artificial Features", IEEE Transactions on Biomedical Engineering, Feb. 2007, Vol. 54, Issue 2, pp. $212-224$

[15] Faul S., Gregorcic G., Boylan G., Marnane W., Lightbody G., Connolly, S., "Gaussian Process Modeling of EEG for the Detection of Neonatal Seizures", IEEE Transactions on Biomedical Engineering, Dec. 2007, Vol. 54, Issue 12, pp. $2151-2162$.

[16] Zeljković V., Druzgalski C., Li Y., Chen J., Yin X., Bojic M., Mayorga P., "Automated Pre-Ictal Phase Detection Algorithm from EEG Signals", Pan American Health Care Exchanges Conference PAHCE, pp. 1-5, Medellin, Colombia, 29 April to 4 May 2013.

[17] Zeljković V., Valev V., Tameze C., Bojic M., "Pre-Ictal Phase Detection Algorithm Based on One Dimensional EEG Signals and Two Dimensional Formed Images Analysis", High Performance Computing \& Simulation conference 2013, International Workshop on Machine Learning, Pattern Recognition and Applications MLPRA 2013, pp. 607-614, Helsinki, Finland, 1-5 July 2013.
[18] http://www.meb.unibonn.de/epileptologie/science/physik/eegdata.html

[19] Andrzejak RG, Lehnertz K, Rieke C, Mormann F, David P, Elger CE, "Indications of nonlinear deterministic and finite dimensional structures in time series of brain electrical activity: Dependence on recording region and brain state", Phys. Rev. E, 64, 061907, 2001, pp. $1-8$.

[20] Chua K. C, Chandran V, Rajendra Acharya, Lim C. M., "Higher Order Spectral (HOS) Analysis of Epileptic EEG Signals", Proceedings of the 29th Annual International Conference of the IEEE EMBS Cité Internationale, pp. 6495-6498, Lyon, France August 23-26, 2007.

[21] Adeli, Hojjat, Ziqin Zhou, and Nahid Dadmehr. "Analysis of EEG records in an epileptic patient using wavelet transform." Journal of neuroscience methods 123.1 (2003): 69-87.

[22] Chang, Chih-Chung, and Chih-Jen Lin. "LIBSVM: a library for support vector machines". ACM Transactions on Intelligent Systems and Technology (TIST) 2.3 (2011): 27.

[23] Tang, Y., and D. M. Durand. "A tunable support vector machine assembly classifier for epileptic seizure detection". Expert systems with applications 39.4 (2012): 3925-3938. 